\title{
Evolving attitudes toward robotic surgery among Canadian urology residents
}

\author{
Jennifer Locke, MD; Michael Robinson, MD; Andrew MacNeily, MD; S. Larry Goldenberg, MD; \\ Peter C. Black, MD
}

Department of Urologic Sciences, University of British Columbia, Vancouver, BC, Canada

Cite as: Can Urol Assoc J 2017;11 (7):E266-70. http://dx.doi.org/10.5489/cuaj.4222

Published online July 11, 2017

\section{Abstract}

Introduction: Robotic-assisted laparoscopic surgery (RAS) has not been adopted as rapidly or widely in Canada as in the U.S. In 2011, Canadian urology residents felt that RAS represented an expanding field that could potentially negatively impact their training. We re-evaluate trainee exposure and attitudes to RAS in Canadian residency training five years later.

Methods: All Canadian urology residents were asked to participate in an online survey designed to assess current resident exposure to and perception of RAS.

Results: The response rate was 39\% (61/157). Seventy-seven percent of residents reported being involved in at least one RAS procedure (52\% in 2011), and the majority had exposure to $<10$ cases. For those in hospitals with access to RAS, 96\% desired more console time, while only $50 \%$ of those without access wanted more console experience. Of all residents, 50\% felt that RAS will become the gold standard in certain urological surgeries (34\% in 2011), but only $28 \%$ felt that RAS would play an increasingly important role in urology (59\% in 2011).

Conclusions: Despite an increase in exposure to RAS in residency programs over the past five years, console experience remains limited. Although these residents desire more access to RAS, many voice uncertainty of the role of RAS in Canada. We cannot conclude whether RAS is perceived by residents to be beneficial or detrimental to their training nationwide. Moving forward in the robotic era, it will be important to either modify residency curricula to address RAS experience or to limit RAS to fellowship training.

\section{Introduction}

Robotic-assisted laparoscopic surgery (RAS) has expanded widely since it was first described in 1985. ${ }^{1}$ Between 2007 and 2010 the number of RAS cases performed globally nearly tripled from 80000 to $205000 .^{2}$ To date, more than 3 million cases have been completed worldwide. Many institutions in Europe and the U.S. have adopted RAS as the gold standard technique for radical prostatectomy, radical cystectomy, pyeloplasty, and partial nephrectomy. ${ }^{3,4}$

The impact of RAS on the training of residents in general urology has become an increasing focus of attention in Europe and the U.S. ${ }^{5}$ In Canada, where there are 80-fold fewer da Vinci surgical systems ${ }^{\mathrm{TM}}$ (Intuitive Surgical, Inc., Sunnyvale, CA, U.S.) than in the U.S., the role of RAS in urological surgery remains unclear, with no directed training strategy for RAS in the 12 Canadian urology residency programs. In 2014, there were 24 clinical da Vinci surgical systems in Canada (one in British Columbia, five in Alberta, 11 in Ontario, and seven in Quebec), with nine in resident training programs (compared to five in 2011). RAS is not considered a core competency within the urology Royal College of Physicians and Surgeons of Canada objectives of training, resulting in non-standardized, highly variable resident training across the country.

In 2011, we surveyed Canadian urology residents and found that they viewed RAS as an expanding field that could potentially negatively impact their general urology training, ${ }^{6}$ but they wanted to acquire more experience in RAS. RAS urology cases in Canada have increased substantially since 2011 ( 1000 cases in 2011 to $\sim 2200$ cases in 2014) so we re-evaluate Canadian residents' exposure to and attitude towards RAS five years later, having hypothesized in 2011 that RAS would become an integral component of residency training by 2016 .

\section{Methods}

All 157 Canadian urology residents in postgraduate year one through five from 12 anglophone and francophone programs were contacted by email and asked to participate in an online survey. Responses were collected over a four-week period beginning in April 2016. Residents were asked to complete the survey regardless of whether their program possessed a robot. Details of the study and consent were clearly described in the invitation email and residents who agreed to participate followed a link to the survey hosted 
on a secure online database. The survey was available for completion over a four-week period. A reminder was sent to all resident two weeks following the initial contact.

The survey was designed to determine current resident exposure and attitude towards RAS. The same survey as in our prior report was used. ${ }^{6}$ For the purpose of this study, RAS was strictly restricted to experiences with the da Vinci surgical system. Survey participation was anonymous and no identifying information was collected. The project was approved by the University of British Columbia Clinical Research Ethics Board (H10-00081).

\section{Results}

Nine of 12 Canadian programs have a da Vinci surgical system. Of 157 residents contacted from 12 programs, 61 $(39 \%)$ responded, which was the same response rate in our 2011 survey involving 10 programs (50/128=39\%). Eightytwo percent of residents had access to a da Vinci surgical system (compared to 64\% in 2011). All other demographic variables are similar to those of 2011 (Table 1).

\section{Resident experience with RAS}

Seventy-seven percent of residents have been involved in at least one robotic procedure (Fig. 1), which represented an increase from $52 \%$ in 2011 . However, of those who were involved in robotic procedures, 32\%, 33\%, and $81 \%$ had less than five cases as an observer, an assistant, and a console operator, respectively (Fig. 2). For residents in programs with a da Vinci surgical system, $46 \%, 58 \%$, and only $2 \%$

\begin{tabular}{lcc}
\hline Table 1. Resident demographics in $\mathbf{2 0 1 1}$ and 2016 \\
\hline $\begin{array}{c}\text { Residents in } \\
\text { 2011, } \mathbf{n}(\%)\end{array}$ & $\begin{array}{c}\text { Residents in } \\
\text { 2016, } \mathbf{n}(\%)\end{array}$ \\
\hline Sex & & \\
Male & $42(84)$ & $51(84)$ \\
Female & $8(16)$ & $10(16)$ \\
Years of training & & \\
1 & $15(30)$ & $15(25)$ \\
2 & $9(18)$ & $12(20)$ \\
3 & $11(22)$ & $14(23)$ \\
4 & $11(22)$ & $11(18)$ \\
5 & $4(8)$ & $9(14)$ \\
Age & & \\
$20-25$ & $0(0)$ & $5(8)$ \\
$26-30$ & $41(82)$ & $36(59)$ \\
$31-35$ & $8(16)$ & $14(23)$ \\
$36-40$ & $0(0)$ & $5(8)$ \\
$>40$ & $1(2)$ & $1(2)$ \\
da Vinci system in program & & \\
Yes & $32(64)$ & $50(82)$ \\
No & $18(36)$ & $11(18)$ \\
\hline
\end{tabular}

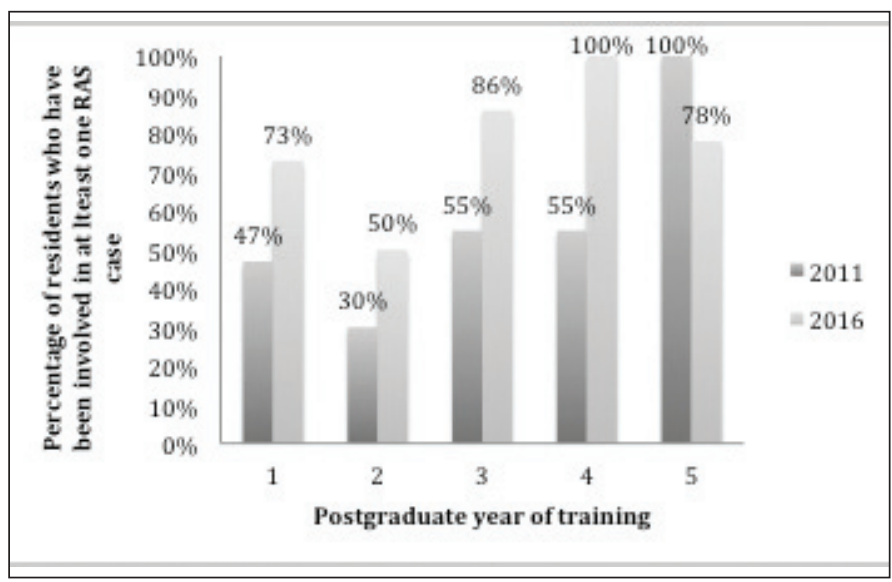

Fig. 1. Resident access to RAS training in 2011 and 2016. Percentage of residents who have been involved in at least one RAS case by year of training.

had "frequent" access as an observer, an assistant, and an operator, respectively.

\section{Resident perceived knowledge and interest in RAS}

The residents rated their own knowledge in RAS as "low," "medium," and "high" in 39\%, 49\%, and 12\%, respectively, which compared to $60 \%, 24 \%$, and $16 \%$ in 2011 (Table 2). Interest was rated as "low," "medium," and "high" by 20\%, $54 \%$, and $26 \%$ of residents, respectively, which compared to $12 \%, 60 \%$, and $28 \%$ in 2011 . Overall, this represents minimal change in interest with a shift in knowledge from "low" to "medium" since 2011. In 2016, 68\% of residents with access to a da Vinci surgical system rated their knowledge as "medium" or "high" and 58\% rated their interest as "medium" or "high." Of those residents without access to a da Vinci surgical system, $73 \%$ rated their knowledge as "low," while $36 \%$ rated their interest as "low." This suggests that those without access have moderate interest, but

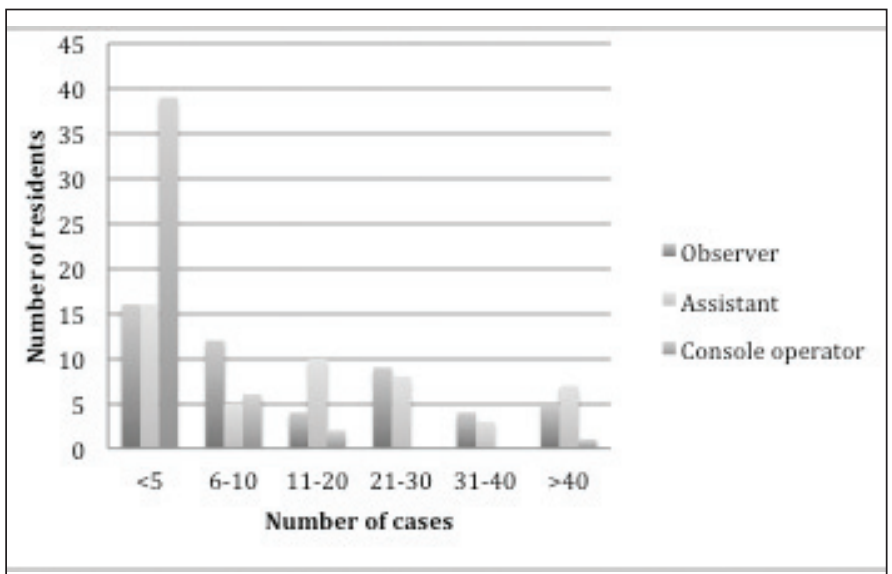

Fig. 2. Resident experience in different roles of RAS in 2016. Number of residents as stratified by number of cases and different roles (observer, assistant, console operator). 
Locke et al.

\begin{tabular}{|c|c|c|}
\hline \multicolumn{3}{|c|}{$\begin{array}{l}\text { What is your level of knowledge with respect to robotic assisted } \\
\text { surgery? }\end{array}$} \\
\hline Answer options & Response in 2011, \% & Response in 2016, \% \\
\hline High & 16.0 & 11.5 \\
\hline Medium & 24.0 & 49.2 \\
\hline Low & 60.0 & 39.3 \\
\hline \multicolumn{3}{|c|}{$\begin{array}{l}\text { What is your current level of interest with respect to robotic } \\
\text { assisted surgery? }\end{array}$} \\
\hline High & 28.0 & 26.2 \\
\hline Medium & 60.0 & 54.1 \\
\hline Low & 12.0 & 19.7 \\
\hline
\end{tabular}

perceive significant limitations in their knowledge. There was little variation in knowledge and interest between junior (PGY1-2) and senior (PGY3-5) residents.

In 2016, the majority (57\%) of residents in programs with a da Vinci surgical system felt that the presence of RAS was beneficial to their training (Table 3). This increased from $27 \%$ in 2011 . In 2016, the majority (61\%) of residents without access to a da Vinci felt that this was beneficial to their training, almost double the $37 \%$ in 2011 . In $2016,96 \%$ of residents with access to RAS desired more console time, while only $50 \%$ of those without access desired more console time (Fig. 3). These trends were similar to those observed in 2011.

\section{Resident perception towards feasibility of RAS}

Several survey questions assessed trainee perception towards the general use of RAS in the future (Table 4). Compared to 2011, fewer residents in 2016 felt that the use of RAS would increase $(61 \%$ in 2016 vs. $92 \%$ in 2011) and that RAS would fulfill an increasingly important role in urology (28\% in 2016 vs. $59 \%$ in 2011). Of those with and without access to RAS, $70 \%$ and $77 \%$, respectively, were uncertain or did not think that RAS would be feasible in Canada. Yet,

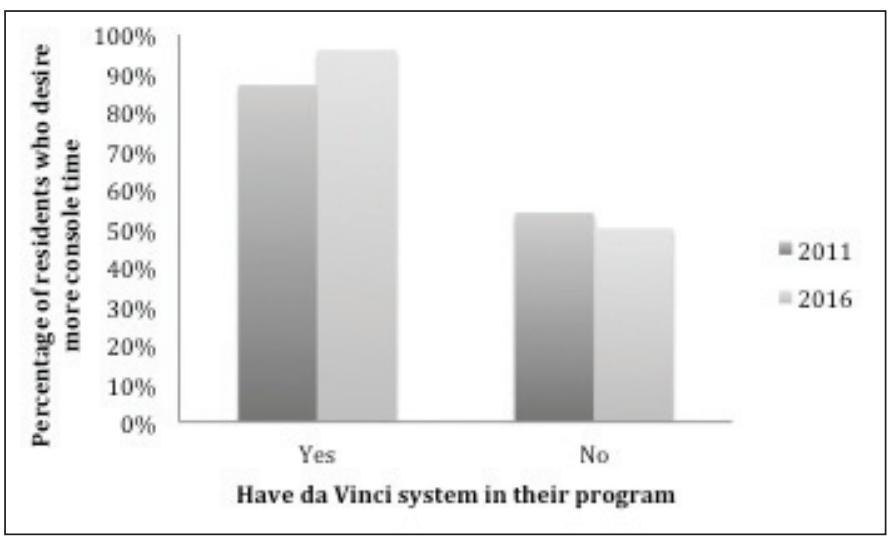

Fig. 3. Resident desire for more console time in 2011 and 2016. Percentage of residents who desire more console time as stratified by access to a da Vinci system in their program.
Table 3. Residents views regarding the influence of having access to a da Vinci surgical system on their training in 2011 and 2016

How do you feel having access to a da Vinci surgical system affects your residency training?

\begin{tabular}{lcc}
\hline Answer options & $\begin{array}{c}\text { Response in } \\
\mathbf{2 0 1 1} \%\end{array}$ & $\begin{array}{c}\text { Response in } \\
\mathbf{2 0 1 6} \%\end{array}$ \\
\hline Beneficial & 26.5 & 57.1 \\
Detrimental & 67.6 & 28.6 \\
Does not affect my training & 5.9 & 14.3 \\
\hline
\end{tabular}

How do you feel not having access to a da Vinci surgical system affects your residency training?

$\begin{array}{lll}\text { Beneficial } & 37.0 & 60.9 \\ \text { Detrimental } & 19.0 & 26.1 \\ \text { Does not affect my training } & 44.0 & 13.0\end{array}$

Do you feel the presence of RAS rduces your case volume for open surgical procedures during residency?

\begin{tabular}{lc}
\hline Answer options & Response in 2016, \% \\
\hline Yes & 82.5 \\
No & 8.8 \\
Unsure & 8.8 \\
\hline Do you feel the presence of RAS reduces your case volume for \\
laparoscopic surgical procedures during residency? \\
\hline Yes & 78.6 \\
No & 12.5 \\
Unsure & 8.9 \\
\hline RAS: robotic-assisted laparoscopic surgery.
\end{tabular}

more of the residents felt that RAS was superior to open and laparoscopic surgical techniques $(49 \%$ in 2016 vs. $24 \%$ in 2011 for open, $56 \%$ in 2016 vs. $36 \%$ in 2011 for laparoscopic). Fifty percent of the residents in 2016 felt that RAS would become the new gold standard for certain surgical procedures in urology as compared to only 34\% in 2011. Of those with and without access to RAS, $47 \%$ and $74 \%$, respectively, felt that it would not become the gold standard for certain surgical procedures.

\section{Resident general opinions regarding RAS}

Open comments were also invited in the survey (Appendix 1). A recurrent concern was the lack of training experience during residency and some residents felt that RAS was detrimental to their learning experience for open radical prostatectomies.

\section{Discussion}

We conducted a survey to evaluate the evolution in attitudes of Canadian urology residents towards RAS between 2011 and 2016.

Our study highlights a widening disparity in resident knowledge and interest between programs with and without access to a da Vinci surgical system. The majority of 


\section{Table 4. Resident views regarding the future of RAS in 2011 and 2016}

What do you feel will happen to the prevalence of robotic assisted surgery?

\begin{tabular}{lcc}
\hline Answer options & Response in 2011, \% & Response in 2016, \% \\
\hline Increase & 92 & 61 \\
Decrease & 2 & 7 \\
Remain unchanged & 6 & 32 \\
\hline
\end{tabular}

Do you feel robotic assisted surgery will fulfill an increasingly important role in urology?

\begin{tabular}{lll} 
Yes & 59 & 28 \\
No & 10 & 41 \\
Unsure & 31 & 31 \\
\hline
\end{tabular}

Do you feel robotic assisted surgery is superior to traditional open surgical techniques?

$\begin{array}{lll}\text { Yes } & 24 & 49 \\ \text { No } & 48 & 36 \\ \text { Unsure } & 28 & 15\end{array}$

Do you feel robotic assisted surgery is superior to laparoscopic surgical techniques?

$\begin{array}{lll}\text { Yes } & 36 & 56 \\ \text { No } & 44 & 16 \\ \text { Unsure } & 20 & 28\end{array}$

Do you feel robotic assisted surgery will become the new gold standard for certain surgical procedures in urology?

\begin{tabular}{lll} 
Yes & 34 & 50 \\
No & 32 & 32 \\
Unsure & 34 & 18 \\
\hline
\end{tabular}

RAS: Robotic-assisted laparoscopic surgery.

those with access report good knowledge, interest, and a general feeling that RAS is beneficial to their training. Those without access have moderate interest, low knowledge and, in contrast, believe that the absence of RAS is beneficial to their training. Nevertheless, $50 \%$ were still interested in accessing console time. This disparity highlights the lack of understanding of robotic surgery at both the level of the trainee and of the training programs across Canada.

Within Europe and the U.S., the role of RAS is established as the standard technique for many urological surgeries., ${ }^{3,4}$ In Canada, complex procedures are performed at both academic and community hospitals, with most of the academic training programs having access to a da Vinci surgical system. Because of funding challenges, smaller community centres and even larger, non-academic hospitals in an urban setting, may never have access to a da Vinci surgical system. So when training residents to meet the healthcare needs of the diverse Canadian population, programs are expected to produce graduates competent to perform general urological surgeries in either the academic or community settings. In our study, the majority of residents agreed that RAS was superior to open and laparoscopic procedures, yet, in those with access, the majority felt that RAS reduced open and laparoscopic case volumes. A particular concern was that
RAS was detrimental to their learning experience for open radical prostatectomies, while at the same time they were not being adequately trained to competently perform RAS independently. It is clearly concerning that graduates may not be competent in key urological surgeries (either by RAS or open techniques) if there is too much emphasis on RAS, decreased open volumes, or inadequate hands-on console training within a program.

The number of RAS cases has almost doubled in three years in Canada and there has been an overall increase in resident access to RAS from $64 \%$ to $82 \%$. Despite these increases, residents continue to have low exposure to RAS as observers, assistants, and importantly, operators on the console. Although we observed more senior residents had increased exposure to RAS as assistants, we also note that this did not translate to time on the console itself. The majority of PGY5s conducted less than five cases on the console.

To keep pace with this continual rise, the questions then become: 1) Do residency programs improve the overall RAS training by sending those without access to a da Vinci surgical system to sites that do have access? and 2) Does the RAS curriculum need to include more hands-on training, or do residents require further fellowship training specifically in the area of RAS in order to perform these surgeries independently? To address this issue a Urology Specialty Committee of the Royal College may have to adapt curricula to include RAS, much like that in the U.S., where the American Urology Association has created a nationwide RAS course/curriculum (http://www.auanet.org/education/educational-programs/elearning/blus-handbook-of-laparoscopic-and-robotic-fundamentals). On the other hand, Moriarty et al suggest that fellowship training in RAS is beneficial, particularly in urology where perioperative outcomes of RAS partial nephrectomy may be better in surgeons who are fellowship-trained. ${ }^{7}$

There are limitations to our study. In contrast to 2011, francophone residents were included in the 2016 survey, although the survey was not translated into French. The addition of programs to the cohort may have contributed to differential responses between 2011 and 2016. Also, in comparing data from 2011 to 2016 to assess for evolving attitudes, we know that the residents surveyed in 2016 are not the same as the residents in 2011. Since the response rate to the survey was only 39\% in both 2011 and 2016, differential sampling may explain some of the different responses between 2011 and 2016. This could explain, for example, that in 2016 only $78 \%$ of PGY5s were involved in RAS, but this number was $100 \%$ in 2011 . This survey addressed only the resident perspective and not the perspective of the trainers and program directors. Their perspectives may provide more insight into the importance of RAS in residency training. Exposure to RAS varied by year of training, which likely contributed to variable responses to questions that depended on RAS exposure. Lastly, this survey was directed 
Locke et al.

at an overall perspective of RAS and did not specify RAS surgeries for specific sites (i.e., prostate, bladder, kidney) and therefore we cannot draw conclusions regarding these different procedures.

\section{Conclusion}

Despite an increase in exposure to RAS in training over the past five years, most residents continue to lack case experience. Accessibility to RAS during residency has been demonstrated to play a significant role in the perception of residents in terms of their knowledge and interest. Moving forward in the robotic era, it will be important to clarify these contradictory perceptions either by modifying residency curricula to address RAS experience or to limit concentrated RAS training to formal fellowships. In Canada, with limited numbers of robotically equipped hospitals, the latter option may be the best.

Competing interests: Dr. Robinson has received grants/honoraria from Astellas and Janssen. Dr. Black has been an advisor for AbbVie, Amgen, Astellas, Bayer, BioCancell, Biosyent, Cubist, Ferring, Janssen, Lilly, Merck, Roche, Sanofi, Sitka, and Spectrum; a speaker for AbbVie, Biosyent, Janssen, and Novartis; has received travel support from Cubist, Janssen, and Sanofi; received collaborative research funding from GenomeDx, iProgen, and New B Innovation; and has been the principle investigator for a clinical trial supported by Roche. The remaining authors report no competing personal or financial interests.
This paper has been peer-reviewed.

\section{References}

1. Leal Ghezzi T, Campos Corleta 0. 30 years of robotic surgery. World I Surg 2016;40:2550-7. https://doi.org/10.1007/s00268-016-3543-9

2. Barbash Gl, Glied SA. New technology and healthcare costs—the case of robot-assisted surgery. N Engl $J$ Med 2010;363:701-4. https://doi.org/10.1056/NEJMp1006602

3. Sood A, Jeong W, Peabody JO, et al. Robot-assisted radical prostatectomy: Inching toward gold standard. Urol Clin North Am 2014;41:473-84. https://doi.org/10.1016/i.ucl.2014.07.002

4. Husain FZ, Badani KK, Sfakianos JP, et al. Emerging surgical treatments for renal cell carcinoma. Future Oncol 2016;12:921-9. hrtps://doi.org/10.2217/fon.15.362

5. Evans $\mathrm{CH}$, Schenarts KD. Evolving educational techniques in surgical training. Surg Clin North Am 2016:96:71-88. https://doi.org/10.1016/i.suc.2015.09.005

6. Robinson $M$, Macneily A, Goldenberg L, et al. Status of robotic-assisted surgery among Canadian urology residents. Can Urol Assoc J 2012;6:160-7. https://doi.org/10.5489/cuaj.11190

7. Moriarty MA, Nepple KG, Tracy CR, et al. Impact of robotic fellowship experience on perioperative outcomes of robotic-assisted laparoscopic partial nephrectomy. Curr Urol 2016;9:19-23. https://doi.org/10.1159/000442845

Correspondence: Dr. Peter Black, Department of Urologic Sciences, University of British Columbia, Vancouver, BC, Canada; pblack@mail.ubc.ca

\footnotetext{
Appendix 1. Resident written comments

While residents in U.S. get more and more console time, here in Canada, RAS is basically not for residents!

We are a high-volume RAS centre. I would argue that RAS is already a gold standard for procedures like prostatectomy.

Surgeon-dependent exposure to upper-level residents only.

Robotic surgery will inevitably become the standard of care for certain procedures, such as radical prostatectomy, in the Western world, and already has at our centre (one surgeon doing open prostates vs. seven doing robotic prostatectomies) and will contribute to the further subspecialization of our specialty, creating "proceduralists" as opposed to surgeons with a broad procedural repertoire. This will be the inevitable consequence of surgical fields, as more and more evidence for the outcomes of high- vs. low-volume surgeon, but the degree of specialization we encourage/endorse will be a matter of a united consensus amongst the urology community. Robotic surgery is a platform that lends itself to this differentiation/specialization given its scarcity in our country and the way we deal with this moving forward will require intentional and goal-directed action by the Royal College and those structuring the curriculum and accreditation of urological training in Canada.

If robot is to be integrated, it needs to be early; start on console in practice, then bedside, then real console time, which is often omitted in programs, even those with a robot.

Biggest problem in our program. Can't do open prostates. Can't do robotics surgery. Taken away from open surgery training
} 